Journal of Chemical Ecology, Vol. 31, No. 6, June 2005 ((C2005)

DOI: $10.1007 / \mathrm{s} 10886-005-5296-\mathrm{z}$

\title{
ERRATUM
}

\section{THE ROLE OF CONTACT PHEROMONES IN MATE LOCATION AND RECOGNITION IN Xylotrechus colonus}

\author{
MATTHEW D. GINZEL, ${ }^{1}$ GARY J. BLOMQUIST, ${ }^{2}$ \\ JOCELYN G. MILLAR, ${ }^{3}$ and LAWRENCE M. HANKS, ${ }^{1, *}$ \\ ${ }^{1}$ Department of Entomology, University of Illinois at Urbana-Champaign, Urbana, \\ IL 61801, USA \\ ${ }^{2}$ Department of Biochemistry and Molecular Biology, University of Nevada, \\ Reno, NV 89557-0014, USA \\ ${ }^{3}$ Department of Entomology, University of California, Riverside, \\ CA 92521, USA
}

* To whom correspondence should be addressed. E-mail: hanks@life.uiuc.edu 
We regret that Table 1 of this article on page 540 contained several errors. A corrected version of the table is as follows:

Table 1. Cuticular Hydrocarbons of Female And Male Xylotrechus colonus ${ }^{a}$

\begin{tabular}{|c|c|c|c|c|}
\hline Peak & Hydrocarbon & Female & Male & Diagnostic ions \\
\hline 1 & $n-\mathrm{C} 25$ & + & + & $352\left(\mathrm{M}^{+}\right)$ \\
\hline 2 & 9-MeC25 & + & - & $140,252 / 253,366\left(\mathrm{M}^{+}\right)$ \\
\hline 2 & 11-MeC25 (trace) & + & - & $168 / 169,224 / 225$ \\
\hline 3 & 2-MeC25 & + & + & $323,351,366\left(\mathrm{M}^{+}\right)$ \\
\hline 4 & 3-MeC25 & + & - & $309,337,366\left(\mathrm{M}^{+}\right)$ \\
\hline 5 & 2-MeC26 & - & + & $337,365,380\left(\mathrm{M}^{+}\right)$ \\
\hline 6 & $n-\mathrm{C} 27$ & + & + & $380\left(\mathrm{M}^{+}\right)$ \\
\hline 7 & $11,13-\mathrm{MeC} 27$ & + & + & $\begin{array}{l}168 / 169,196 / 197,224 / 225,252 / 253,394 \\
\left(\mathrm{M}^{+}\right)\end{array}$ \\
\hline 8 & 2-MeC27 & + & + & $351,379,394\left(\mathrm{M}^{+}\right)$ \\
\hline 9 & 3-MeC27 & + & + & $337,365,394\left(\mathrm{M}^{+}\right)$ \\
\hline 10 & $n-\mathrm{C} 28$ & + & + & $394\left(\mathrm{M}^{+}\right)$ \\
\hline 11 & 13-MeC28 & + & + & $196 / 197,238 / 239$ \\
\hline 11 & 12,11-MeC28 (trace) & + & + & $168 / 169,182 / 183,252 / 253,266 / 267$ \\
\hline 12 & $\mathrm{C} 29: 1$ & + & + & $406\left(\mathrm{M}^{+}\right)$ \\
\hline 13 & $\mathrm{C} 29: 1$ & + & - & $406\left(\mathrm{M}^{+}\right)$ \\
\hline 14 & 3-MeC28 & - & + & $351,379,408\left(\mathrm{M}^{+}\right)$ \\
\hline 15 & $n-\mathrm{C} 29$ & + & + & $408\left(\mathrm{M}^{+}\right)$ \\
\hline 16 & $11,13,15-\mathrm{MeC} 29$ & + & + & $\begin{array}{l}168 / 169,196 / 197,224 / 225,252 / 253, \\
280 / 281,422\left(\mathrm{M}^{+}\right)\end{array}$ \\
\hline 17 & $\mathrm{C} 31: 1$ & - & + & $434\left(\mathrm{M}^{+}\right)$ \\
\hline
\end{tabular}

${ }^{a}$ Peak numbers correspond with those in Figure 1. "+" indicates a compound is present and "-" indicates it is absent. 11-MeC25 and 12,11-MeC28 coeluted in trace amounts with other compounds. Peaks 12 and 13 represent isomers of the same alkene. 